\title{
Fallbericht 39-jährige III. Gravida, III. Para mit Inversio uteri nach Steißgeburt
}

\section{Case report of 39-Year-Old Gravida III, Para III with Inverted Uterus after Breech Birth}

Autor

Sebastian Findeklee

Institut

Universitätsklinik für Frauenheilkunde, Geburtshilfe und

Reproduktionsmedizin, Universitätsklinikum Magdeburg, Magdeburg

Schlüsselwörter

Inversio uteri, Steißgeburt, manuelle Reposition

Key words

inversio uteri, breech birth, manual reposition

$\begin{array}{ll}\text { eingereicht } & 18.11 .2018 \\ \text { angenommen } & 19.12 .2018 \\ \text { nach Überarbeitung }\end{array}$

Bibliografie

DOI https://doi.org/10.1055/a-0828-8696

Online-Publikation: 4.2.2019

Z Geburtsh Neonatol 2020; 224: 38-41

(c) Georg Thieme Verlag KG Stuttgart · New York

ISSN 0948-2393

Korrespondenzadresse

Dr. Sebastian Findeklee

Universitätsklinik für Frauenheilkunde, Geburtshilfe und

Reproduktionsmedizin

Universitätsklinikum Magdeburg

Gerhart-Hauptmann-Straße 35

39108 Magdeburg

Tel.: + 49/391/6717 399, Fax: + 49/391/6717 311

sebastian.findeklee@arcor.de

\section{ZUSAMMENFASSUNC}

Die Inversio uteri bezeichnet das Umstülpen der Gebärmutter in die Scheide postpartal. Sie ist äußerst selten, aber stellt einen geburtshilflichen Notfall dar. Die Gefahr für die Gebärende besteht in einer Uterusatonie mit dem Verlust großer Mengen Blut in kurzer Zeit. Dies kann, wenn keine rechtzeitige Therapie erfolgt, zum hypovolämischen Schock bis zum Herz-Kreislaufversagen führen. Die Therapie erfolgt durch eine Uterus-Reposition in Vollnarkose mit anschließender manueller Plazentalösung. Anschließend folgen die Kürettage und die Gabe von hochpotenten Uterotonika wie Sulproston. Durch den in der Regel hohen Blutverlust kommt es häufig zu einer Störung des Gerinnungssystems, sodass eine Substitution von Antifibrinolytika wie Tranexamsäure, Gerinnungsfaktoren und/oder Erythrozytenkonzentraten erforderlich wird. Gelingt keine vaginale Reposition des Uterus, so bleiben als Ultima Ratio nur die Reposition per Laparotomie oder die Hysterektomie. Wir berichten über eine 39-jährige III. Gravida, III. Para mit 36+3 Schwangerschaftswochen (SSW), bei der es nach spontaner Steißgeburt bei Placenta accreta zur Inversio uteri kam, die vaginal reponiert werden konnte.

\section{ABSTRACT}

An inverted uterus is defined as the eversion of the uterus into the vagina. It is a very rare event but has to be treated as an emergency. A woman with an inverted uterus is in serious danger of developing atony with high blood loss in a short time. This can lead to hypovolemic shock and circulatory failure. Therapy includes urgent uterine repositioning under narcosis and curettage after manual abruption of the placenta. Afterwards, uterotonics such as prostaglandins should be administered. A disorder of the coagulation system is very common due to blood loss. Therefore, substitution of plasma components and antifibrinolytic drugs are recommended. If vaginal repositioning of the uterus cannot be achieved, laparotomy or hysterectomy are possible alternatives. We report the case of a 39 year-old gravida III, para III in the $36+3$ gestational week who, after a spontaneous breech delivery with placenta accreta, experienced an inverted uterus, which was then repositioned.

\section{Einleitung}

Inversio uteri bezeichnet die komplette oder inkomplette Umstülpung der Gebärmutterschleimhaut in die Scheide oder bis zur Vulva. Sie tritt bei ca. 1:10 000 Geburten auf [1]. Maternale Risiko- faktoren sind die fetale Makrosomie, Mehrlingsgeburten, Multiparität, Bindegewebsschwäche, Plazentationsstörungen, höheres Alter oder ein Uterus myomatosus. Geburtshilfliche Risikofaktoren sind der forcierte Nabelschnurzug in der Plazentarperiode (cord 
traction), der Credé-Handgriff, ein protrahierter Geburtsverlauf oder vorangegangene operative Eingriffe an der Gebärmutter $[2,3]$. Hauptrisikofaktor ist die iatrogene Inversion durch forcierte Manöver der Plazentaentwicklung bei abnorm invasiver Plazenta. Pathophysiologisch kommt es zu einem Umstülpen des Uterus an der plazentaren Haftstelle der Nabelschnur. Der Isthmus uteri am Übergang zur Zervix wirkt als Schnürring und hält den invertierten, in sich gestülpten Uterus in dieser Position fest [4]. Hinsichtlich des zeitlichen Auftretens post partum unterscheidet man die akute (innerhalb von $24 \mathrm{~h}$ ), die subakute (nach mehr als $24 \mathrm{~h}$ ) und die chronische (nach mehr als 1 Monat) Inversion des Uterus. \ Tab. 1 zeigt die verschiedenen Schweregrade der Uterus-Inversion. Klinisch äuBert sich die Inversio uteri infolge Zugs am Peritoneum durch plötzlich einsetzende stärkste Schmerzen bei sichtbar umgestülptem, im Bereich der Scheide sichtbarem Uterus. Aufgrund einer Atonie des Uterus kommt es innerhalb kürzester Zeit zu einem ausgeprägten Blutverlust und es droht ein hypovolämischer Schock mit disseminierter intravasaler Gerinnung [5]. Therapeutisch wird die sofortige Reposition des Uterus in Narkose nach Johnson erforderlich. Hierbei kommt es zunächst zu einer manuellen Plazentalösung. Anschließend wird der Uterus manuell komprimiert. Danach wird der Uterus in seine ursprüngliche anatomische Position zurückgeschoben. Ist diese gelungen, sollten Uterotonika verabreicht werden, um den Uterus in seiner Lage zu halten [6]. Gelingt die Reposition nicht über die Scheide, kommt der Repositions-Versuch per Laparotomie in Frage. Hierbei wird der Uterus in seine normale anatomische Lage geschoben (sogenanntes Huntington-Manöver), wenn eine vaginale Reposition z. B. aufgrund eines ausgeprägten zervikalen Schnürrings nicht gelingt. In manchen Fällen einer Inversio uteri erfolgt als Ultima ratio die notfallmäßige Hysterektomiebevorzugt suprazervikal-, wenn es durch alle anderen Maßnahmen nicht zu einem Sistieren der Blutung kommt. In jedem Fall muss in der Folge eine Substitution des verlorenen Blutvolumens und der Gerinnungsfaktoren erfolgen [7].

\section{Fallbericht}

Wir berichten über eine 39-jährige III. Gravida, III. Para (Zustand nach $2 \times$ Spontanpartus 2001 und 2004), die sich am 22.12.2014 mit $36+2$ SSW wegen eines gegen 12 Uhr aufgetretenen frühen vorzeitigen Blasensprungs im Kreißsaal vorstellte.

\section{Anamnese}

Die Gebärende hatte sich während der Schwangerschaft bereits zweimal in der 20. und 23. SSW wegen einer präpartalen Blutung bei Verdacht auf Placenta praevia vorgestellt. Sonst gestaltete sich

- Tab. 1 Schweregrade der Inversio uteri.

\begin{tabular}{|l|l|}
\hline Schweregrad & klinische Charakteristika \\
\hline Grad 1 & Fundus ragt in das Cavum uteri \\
\hline Grad 2 & Fundus befindet sich auf Höhe der Tubenostien \\
\hline Grad 3 & Fundus fällt in die Scheide vor \\
\hline Grad 4 & komplette Inversion \\
\hline
\end{tabular}

der Schwangerschaftsverlauf unauffällig. Das Ersttrimesterscreening ergab einen unauffälligen Befund.

\section{Klinischer Verlauf prä- und subpartual}

Bei der Aufnahmeuntersuchung am Nachmittag war die Portio $1 \mathrm{~cm}$ erhalten und der Muttermund bei Abgang von reichlich klarem Fruchtwasser $1 \mathrm{~cm}$ eröffnet. Sonografisch zeigte sich ein zeitgerecht entwickelter Fet mit einem Schätzgewicht von $2835 \mathrm{~g}$ in II. Beckenendlage. Nach ausführlicher Aufklärung über das Für und Wider der beiden Entbindungsmodi Spontanpartus und Sectio caesarea wünschte die Frau die Spontangeburt. Wir nahmen die Gebärende stationär auf und besprachen zunächst das Abwarten eines spontanen Wehenbeginns. Nachdem es um Mitternacht noch nicht zu einem Wehenbeginn gekommen war und sich der Muttermundsbefund idem im Vergleich zur Aufnahmeuntersuchung zeigte, wünschte die Patientin nun die Geburtseinleitung mit Misoprostol 50 Mikrogramm oral im off label-use. Ca. 40 Min nach der Misoprostol-Gabe entwickelte die Patientin regelmäßige Wehen. Es kam im Verlauf zu einer regelrechten Muttermundseröffnung. Um 03:06 Uhr war der Muttermund dann vollständig eröffnet. Die Patientin wurde im Vierfüßlerstand gelagert. Um 03:18 Uhr begann die aktive Pressperiode. 03:35 Uhr erfolgte der komplikationslose Spontanpartus eines weiblichen Neugeborenen mit einem Gewicht von $2800 \mathrm{~g}$ und einer Länge von $49 \mathrm{~cm}$ (APGAR 9/10/10, Nabelarterien-pH 7,31, Base Excess - 7,8 mmol/I) aus vollkommener Steißlage im Vierfüßlerstand. Das Cardiotokogramm war bis auf wehensynchrone Spikes in den letzten 10 Min der Pressperiode stets unauffällig.

\section{Klinischer Verlauf postpartal}

35 Minuten nach der Geburt zeigte sich die Plazenta noch immer trotz leichtem Zug an der Nabelschnur adhärent. Trotz konservativer homöopathischer Maßnahmen und aktivem Mitschieben bei mäßiger cord traction waren auch 43 Min nach der Geburt die Plazentalösungszeichen negativ. 52 Min postpartal verspürte die Patientin wieder Wehen und schob aktiv mit. 04:27 Uhr kam es dann zur vermeintlichen Geburt der Plazenta. Es zeigte sich jedoch sofort eine vollständige Inversio uteri. Die Plazenta erschien weiterhin komplett dem Uterus adhärent. Es erfolgte die sofortige Indikationsstellung zur Reposition und Plazentalösung in Intubationsnarkose. Auf der Fahrt in den Kreißsaal-OP imponierten die Eihäute auf einer Fläche von $20 \times 25 \mathrm{~cm}$ komplett blutgefüllt. Im Rahmen der Umlagerung der Patientin im Operationssaal kam es zu einer überregelstarken Blutung. Durch die Anästhesie wurde ein zweiter großlumiger venöser Zugang gelegt und über diesen Kreuzblut abgenommen. Anschließend erfolgte um 04:45 Uhr die manuelle Plazentalösung. Bei der Eröffnung der Eihäute entleerten sich 1,5 I Blut. Es zeigte sich eine Placenta accreta. Danach reponierten wir den Uterus vaginal und führten eine Nachkürettage unter sonografischer Kontrolle durch. Zudem wurde ein Dammriss ${ }^{\circ}$ versorgt.

Postoperativ erfolgte eine Tonisierung des Uterus mit Sulproston 500 Mikrogramm in einer $250 \mathrm{ml}$-Infusion. Der intraoperative Hämoglobin-Wert betrug 6,4 g/dl. Der Patientin wurden postoperativ 2 Erythrozytenkonzentrate transfundiert. Bereits intraoperativ erhielt die Patientin $2 \mathrm{~g}$ Tranexamsäure intravenös. Die Patientin blieb nach dem operativen Eingriff noch $12 \mathrm{~h}$ zur Observatio im 
Wachsaal. Sie war stets kreislaufstabil. Der Hämoglobinwert nach Transfusion betrug 10,1 g/dl. Wir verordneten eine intravenöse antibiotische Therapie mit Cefuroxim $3 \times 1500 \mathrm{mg}$ und Metronidazol $2 \times 500 \mathrm{mg}$ für 3 Tage. Die Entlassung von Mutter und Kind erfolgte am 4. postpartalen Tag. Bei der Wiedervorstellung der Patientin einen Monat postpartal berichtete diese über subjektives Wohlbefinden. Im Rahmen der Untersuchung zeigte sich ein unauffälliger Befund ohne Anhalt für einen Prolaps. Die Frage nach einer Störung der Kontinenz wurde von der Patientin verneint.

\section{Diskussion}

Bei der Inversio uteri unserer geburtshilflichen Patientin handelte es sich um einen vitalen Notfall. So kam es zu einem geschätzten Blutverlust von über 1,5 I infolge einer kontinuierlichen Blutung des atonen Uterus in den Zwischenraum zwischen Uterus und Eihäuten. Durch den drohenden hypovolämischen Schock kann es zu einem Herz-Kreislauf-Versagen und infolge einer disseminierten intravasalen Gerinnung zu einem Verbrauch von Gerinnungsfaktoren mit Hyperfibrinolyse kommen. Deshalb verabreichten wir noch während der Operation das Antifibrinolytikum Tranexamsäure, das mittlerweile Eingang in das Management der postpartalen Hämorrhagie unterschiedlicher Genese gefunden hat [8]. Zudem erfolgte eine Transfusion von 2 Erythrozytenkonzentraten. Risikofaktoren unserer Patientin für das Auftreten der Inversio uteri waren das Vorliegen einer Plazentationsstörung und ggf. das mütterliche Alter > 35 Jahre. Von geburtshilflicher Seite lagen keine Risikofaktoren vor. Es kam weder zu einem protrahierten Geburtsverlauf (Eröffnungsperiode, Austreibungsperiode) noch wurde ein forcierter Nabelschnurzug angewendet. Dies erscheint insofern nicht verwunderlich, dass sich eine Uterus-Inversion häufig spontan und unerwartet ereignet [9]. In unserem Fall fiel die Diagnosestellung aufgrund der klinisch deutlich sichtbaren Umstülpung der Gebärmutter nicht schwer und erfolgte ohne zeitliche Verzögerung. In Fällen einer inkompletten Inversio uteri kann die Diagnosestellung jedoch erschwert sein und setzt ein hohes Maß an klinischer Erfahrung voraus. Ein trotz Wehentätigkeit von abdominal nicht tastbarer Fundus sollte den Verdacht auf eine Uterus-Inversion lenken. Bei der Spekulumeinstellung imponiert blutiges Gewebe im Bereich des hinteren Scheidengewölbes. Im konkreten Fall entschieden wir uns zunächst für die manuelle Plazentalösung und dann für die Reposition des Uterus in Vollnarkose. Vorteil dieses Vorgehens war die leichtere Reponierbarkeit der Gebärmutter. Kritisch bei diesem Vorgehen muss betrachtet werden, dass es zu einem Zeitverlust kommt, wodurch es im Falle einer Atonie zu einem beträchtlichen Blutverlust kommen kann. Daher empfehlen einige Autoren zunächst die Reposition und anschließend die Nachkürettage [10]. Als Repositionsmethode der Wahl gilt die manuelle Reposition nach Johnson, bei der die prolabierte Gebärmutter mit dem Daumen und den übrigen 4 Fingern gegriffen und über die Scheide zurückgestülpt wird [6]. Gelingt dies nicht, bleibt nur der abdominale Repositionsversuch über eine Laparotomie. Hierbei werden Klemmen in das Grübchen des Uterus gesetzt und man zieht den Uterus nach kranial, indem die Klemmen schrittweise in Richtung Fundus versetzt werden[11]. Sollte dieser auch frustran sein, muss die Hysterektomie erwogen werden, um die Mutter vor dem drohenden
Herz-Kreislauf-Versagen zu bewahren. Alternativ zu den operativen Methoden der Reposition schlug eine japanische Arbeitsgruppe vor 2 Jahren auch bei Inversio uteri den Einsatz eines Bakri-Ballonkatheters vor [12]. Neben der vitalen Gefahr durch die Blutung muss berücksichtigt werden, dass die Inversio uteri für die betroffene Frau ein extrem schmerzhaftes Ereignis darstellt. Durch massiven Zug am Peritoneum treten stärkste Schmerzen auf. Deshalb sollte vor allen therapeutischen Manövern auf eine suffiziente Schmerztherapie und das rasche Einleiten einer Narkose geachtet werden. Ausreichende Daten über die Betreuung von Frauen mit postpartal aufgetretener Inversio uteri für die Zeit des Wochenbetts oder Möglichkeiten der Rezidivprophylaxe liegen nicht vor. Wichtig erscheint ein regelmäßiges Training des Beckenbodens durch z. B. Beckenbodengymnastik. Daten über die Rezidivhäufigkeit liegen aufgrund der Seltenheit des Ereignisses nicht vor.

\section{FAZIT}

Die Inversio uteri ist ein seltenes, dafür aber akut lebensbedrohliches Ereignis in der Geburtshilfe. Sie erfordert ein schnelles Handeln in Form einer Reposition des Uterus in Narkose, um schwerwiegende Folgekomplikationen für die Mutter wie einen Volumenmangelschock oder eine disseminierte intravasale Gerinnung zu verhindern. Da sich die maternalen Risikofaktoren kaum beeinflussen lassen, sollte das Hauptaugenmerk bei der Verhinderung dieses Ereignisses auf das Vermeiden geburtshilflicher Risikofaktoren liegen. Dazu gehören die kritische Indikationsstellung zum Nabelschnurzug in der Plazentarperiode und das Meiden des forcierten Credé-Handgriffs.

\section{Interessenkonflikt}

Die Autoren geben an, dass kein Interessenkonflikt besteht.

Literatur

[1] Stauber M, Weyerstahl T. Duale Reihe Gynäkologie und Geburtshilfe. 3. Aufl. Stuttgart: Thieme; 2007. doi:10.1055/b-002-6232

[2] Kainer F. Facharzt Geburtsmedizin. 1. AuflMünchen/Jena: Urban und Fischer; 2006 S. $965 f$

[3] Shirota $\mathrm{K}$, Ota T, Tsujioka $\mathrm{H}$ et al. Uterine inversion due to a leiomyoma on postpartum day 41: a case report. J Obstet Gynaecol Res 2011; 37: 897-900. doi:10.1111/j.1447-0756.2010.01420

[4] Blees M, Salfelder A, Upleger F. Puerperal uterine inversion (report of 3 cases). Zentralbl Gynäkol 1994; 116: 48-51

[5] Lober R, Reiher H. Inversio uteri post partum. Zentralbl Gynäkol 1981; 103: $110-114$

[6] Pschyrembel W, Dudenhausen JW. Praktische Geburtshilfe. 16. Aufl. Berlin: de Gruyter-Verlag; 1989 S. 272

[7] van Vugt PJ, Baudoin D, Blom VM et al. Inversio uteri puerperalis. Acta Obstet Gynecol Scand 1981; 60: 353-362 
[8] Sentilhes L, Merlot B, Madar H et al. Postpartum haemorrhage: prevention and treatment. Expert Rev Hematol 2016; 9: 1043-1061. doi:10.1080/17474086.2016.1245135

[9] Rath W, Gembruch U, Schmidt S. Geburtshilfe und Perinatalmedizin. 2. Aufl. Stuttgart: Thieme; 2010. doi:10.1055/b-002-10339

[10] Edis AW. Reposition of Complete Inversion of Uterus after Five Years' Duration. Br Med J 1883; 1: 561-562
[11] Leal RF, Luz RM, de Almeida JP et al. Total and acute uterine inversion after delivery: a case report. J Med Case Rep 2014; 8: 347. doi:10.1186/1752-1947-8-347

[12] Ida A, Ito K, Kubota Y et al. Successful reduction of acute puerperal uterine inversion with the use of a bakri postpartum balloon. Case Rep Obstet Gynecol 2015; 2015: 424891. doi:10.1155/2015/424891 